\title{
A Computational Agent Model Incorporating Prior and Retrospective Ownership States for Actions ${ }^{1}$
}

\author{
Jan Treur \\ VU University Amsterdam, Agent Systems Research Group \\ De Boelelaan 1081, 1081 HV, Amsterdam, The Netherlands \\ treur@cs.vu.nlｈttp://www.cs.vu.nl/ treur
}

\begin{abstract}
The computational agent model presented in this paper generates prior and retrospective ownership states for an action based on principles from recent neurological theories. A prior ownership state is affected by prediction of the effects of a prepared action, and exerts control by strengthening or suppressing actual execution of the action. A retrospective ownership state depends on whether the sensed consequences of an executed action cooccur with the predicted consequences, and is the basis for acknowledging authorship of actions, for example, in social context. It is shown how a number of known phenomena can occur. For example, scenarios were shown for vetoing a prepared action due to unsatisfactory predicted effects, and for mirroring an observed action performed by another agent, without imitating the action. Moreover, it is shown how poor action effect prediction capabilities can lead to reduced retrospective ownership states, as in persons suffering from schizophrenia. The obtained computational model can be used as a basis for simulationbased training, for example, to develop a virtual patient based on the model so that a psychiatrist or psycho-therapist (e.g., during his or her education) can gain insight in the processes in certain types of patients, or to analyse how effective a certain form of therapy can be. A second type of application is in the area of gaming or virtual stories in which, for example, persons with deviations in ownership states play a role and based on that show unexpected behaviour.
\end{abstract}

\section{Introduction}

In the cognitive and neurological literature the notion of ownership of an action has received much attention: in how far does a person attribute an action to him or herself, or to another person. For example, persons suffering from schizophrenia may easily attribute self-generated actions to (real or imaginary) other persons. One of the issues that plays an important role both in the execution decisions for an action, and in its attribution, is the prediction of the (expected) effects of the action, based on internal simulation starting from the preparation of the action (e.g., Wolpert, 1997; Haggard, 2008). If these predicted effects are satisfactory, this may entail a 'go' decision for

\footnotetext{
1 A preliminary, shorter version of this paper was included in Proc. of the Twenty-Second International Joint Conference on Artificial Intelligence, IJCAI'11, 2011; see http://www.few.vu.nl/ wai/Papers/IJCAI11 ownership.pdf
} 
the execution of the action, thus exerting control over action execution. In contrast, less satisfactory predicted effects may lead to vetoing a prepared action: a 'no go' decision.

Predicted action effects play an important role in attribution of the action to an agent after it has been performed. In neurological research it has been found that poor predictive capabilities are a basis for false attributions of actions, for example, for patients suffering from schizophrenia; (e.g., Synofzik et al., 2010; Voss et al., 2010). The traditional approach is that comparison of predicted sensory effects and sensed actual effects (after execution of the action) is an important condition for proper retrospective self-attribution of a self-generated action. The so-called 'comparator model' (e.g., Feinberg, 1978; Frith, 1992; Wolpert, 1997; Frith et al., 2000) is a computational formalisation of this idea. In contrast to this, it has been reported more recently that the predicted sensory effect and the sensed actual effect are not simply compared, but integrated with each other (e.g., Moore and Haggard, 2008; Synofzik et al., 2010; Voss et al., 2010).

The computational agent model presented in this paper aims at providing a computational formalisation for the perspective reported, for example, in the latter recent literature. In designing this computational model, in line with this literature a distinction was made between prior ownership states, among others based on prediction of sensory effects of a prepared action, and retrospective ownership states, for which in addition the monitored execution of the action and the sensed actual effects are used. Prior ownership states play an important role in controlling the actual execution of actions (go/no-go decisions, vetoing), whereas retrospective ownership states are important for acknowledging authorship of an action in a social context, but also may play a role in reflection on one's own functioning and personal learning and development (e.g., learning from less optimal choices).

The computational model is specified at a functional cognitive modelling level, abstracting from more specific neurological details. It can be used as a basis for development of a virtual patient that can be used by therapists, for example during their education to increase their insights in the dynamics of processes involving ownership, and deviations that may occur in these processes. Moreover, in the area of gaming or (agent-based) virtual stories the model can be used to develop virtual characters that show special behaviour in relation to certain characteristics concerning ownership.

In this paper, in Section 3 the computational agent model is presented, based on neurological principles discussed in Section 2. Section 4 illustrates the model by presenting four different scenarios, among which vetoing an action because of unsatisfactory predicted effects, execution of an action with false attribution due to poor predictive capabilities as happens in schizophrenia, and mirroring an observed action of another agent and properly attributing it to the other agent. In Section 5 it is discussed how elements of the model can be related to recent neurological findings. Finally, Section 6 is a discussion.

\section{Neurological Background}

The idea developed over the years is that co-occurrence of predicted sensory effects and sensed actual effects (after execution of the action) is an important condition for proper retrospective selfattribution of a self-generated action (e.g., Moore and Haggard, 2008). In what is called the comparator model or the central monitoring theory (e.g., Feinberg, 1978; Wolpert, 1997; Frith, 1992; Frith et al., 2000; David et al., 2008), the predicted effect and the sensed actual effect are compared or matched. In Figure 1 a schematic overview of the comparator model is shown, comparable to similar pictures shown in (Wolpert, 1997) and (David et al., 2008). 


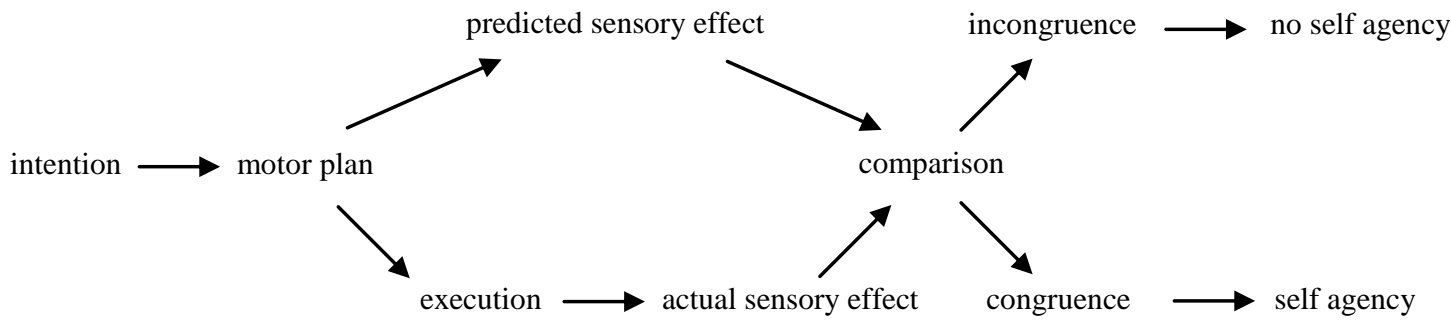

Figure 1 Overview of the comparator model

The notion of comparison was originally taken from the area of cybernetics, and assumes that two activation strengths are compared. For example, if a binary case is assumed, two signals 1 provide an outcome 0 (i.e., no difference: self agency), and a combination of 0 and 1 gives an outcome 1 (no self agency). Also two strengths 0 would give a comparison outcome 0 (self agency) in this model. Moreover, for the nonbinary case, for example, two equal values 0.3 (or 0.5 ) would give an outcome 0 as well, which is the same as the outcome for two values 1 (self agency). Such simple examples, especially for lower but equal values may not always be considered intuitive.

In recent years it has been reported in neurological literature that the predicted effect and the sensed actual effect are not simply compared or matched, as claimed in the comparator model, but in fact are added to each other in some integration process (e.g., Moore and Haggard, 2008; Synofzik et al., 2010; Voss et al., 2010). In such a case the outcome would not always be the same as for the comparator model. For example, integrating two equal levels $l$ will provide an outcome (self agency) that is quite different from the outcome for two equal lower values 0.3 or two equal values 0 . Moreover, the integration process involves a temporal element which is not taken into account in the comparator model: first the predicted sensory effect starts to become active whereas later on the sensing of the actual effect in some sense is added to it.

Furthermore, another peculiar aspect that plays a role here is that within the process, based on the predicted sensory effect suppression of the sensed actual effect takes place, which has been reported since a longer time, for example, around the question 'Why can't you tickle yourself?'; e.g., (Weiskrantz, Elliot, and Darlington, 1971; Claxton, 1975; Collins et al., 1998; Blakemore et al., 1999; Blakemore et al., 2000a; 2000b; Fourneret et al., 2002; Blakemore and Frith, 2003). This shows an interesting phenomenon. On the one hand the predicted effect suppresses the sensed effect, but on the other hand the two effects are combined with each other to obtain a basis for a (retrospective) proper attribution of the action. From a logical point of view these findings from the neurological literature may sound paradoxal, but from a dynamical systems perspective this may well be modelled adequately in a temporal manner, thus providing a more realistic type of model than would be possible in a purely logical setting.

Another element, put forward in (Moore and Haggard, 2008), is the distinction between ownership based on prediction (prior to execution), and ownership based on inference after execution of the action (in retrospect):

'Our results suggest that both predictive and inferential processes contribute to the conscious awareness of operant action. The relative contribution of each of these processes seems to be context dependent. When we can predict the consequences of our actions, as in a high actioneffect contingency block, the awareness of action reflects these predictions. This would provide us with a predictive sense of our own agency. In addition, our results show clear evidence that inferential processes also influence the conscious awareness of operant action.' (Moore and Haggard, 2008, p. 142) 
Also here it is put forward that the temporal pattern may be interesting to take into account:

'The interaction between predictive and inferential processes is of particular interest. (...) The time course over which information about action is built up may be an important clue to this interaction. Specifically, predictive information about actions and effects may operate only over the brief timescale of motor preparation, and may be discarded when sensory evidence makes inference possible. Predictions may be based on an internal forward model (Blakemore et al., 2002). This representation is available for the control of action and on-line guidance of behaviour, but does not outlast the current action. Sensory feedback provides more precise evidence about actions and their effects. This evidence becomes available only after a short sensory delay, but can then be transferred to memory. Thus, reliable and enduring sensory evidence replaces short-lived predictive estimates. We suggest that awareness of action therefore switches from a predictive to an inferential source as the action itself occurs, and as sensory information becomes available. This time-varying mixture of predictive and inferential information may ensure that our experience of our own action is an optimal reflection of the actual relation between our voluntary motor system and the outside world.' (Moore and Haggard, 2008, pp. 142-143)

The issues and perspectives briefly reviewed above have been used as a basis for the computational agent model presented below. More specifically, the following have been taken as a point of departure:

(1) action effect prediction from preparation of an action a to sensory representation of effect b

(2) suppressing the sensory representation of effect $\mathrm{b}$ after this effect was predicted and action a was initiated

(3) a prior ownership state depends on preparation for the action, predicted effects, and context

(4) a retrospective ownership state depends on a combination of predicted sensory effects of the action and action effects sensed afterwards (a form of integration)

(5) a prior ownership state exerts control over the execution of a prepared action (go/no-go decision, vetoing)

(6) a retrospective ownership state is an internal state that also can lead to acknowledging authorship of the action, for example, in social context

In Section 6 it will be discussed in some more detail how the concepts used in the model may relate to neurological concepts.

\section{The Computational Agent Model}

The computational agent model presented below has been designed with the issues discussed in Section 2 as a point of departure (in particular (1) to (6)). For a graphical overview, see Figure 2; here circles denote states (explained in Table 1), and arrows denote which states affect each other. In the model s denotes a stimulus, $c$ a context, a an action, and b a world state affected by the action. Examples of contexts are another agent $B$ which is observed, or the agent self. The effect state $b$ is considered to be positive for the agent (e.g., in accordance with a goal). Note that these are used as parameters in the names of states so that a structured naming convention is obtained; they are not values. The state properties used in the model are summarised in Table 1. Sensor states $\mathrm{ss}(\mathrm{W})$ for a world propert $\mathrm{W}$ are assumed to be generated (i.e., get nonzero activation values) by sensing or observing the world. 


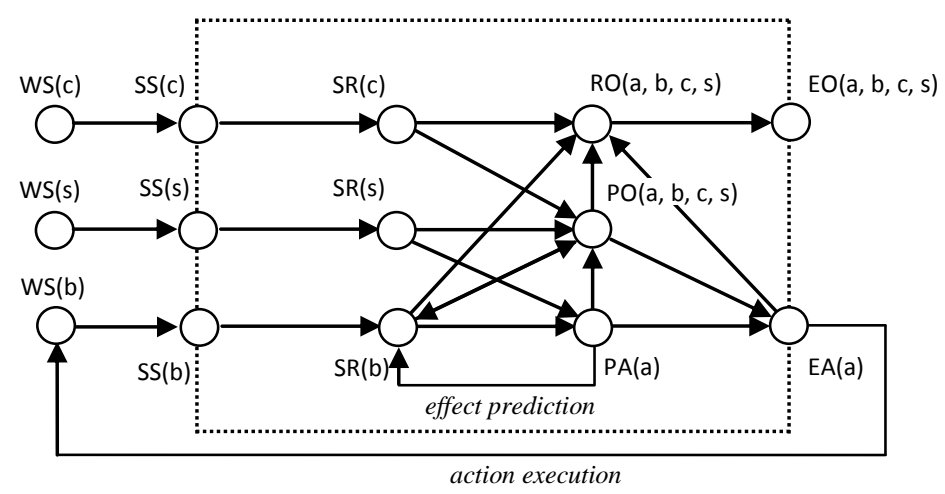

Figure 2 Overview of the computational agent model

This includes the context $\mathrm{c}$ which can be self or another agent B. As expressed in (3) and (4) in Section 2, the computational agent model distinguishes prior and retrospective ownership states for actions, indicated by $\mathrm{PO}(\mathrm{a}, \mathrm{b}, \mathrm{c}, \mathrm{s})$ and $\mathrm{RO}(\mathrm{a}, \mathrm{b}, \mathrm{c}, \mathrm{s})$, respectively (see Figure 2). These states are taken specific for a given action $a$, effect $b$, context $c$, and stimulus s (triggering preparation of a). When the context $\mathrm{c}$ is self, an ownership state for $\mathrm{c}$ indicates self-ownership attribution, whereas for context $\mathrm{c}$ an observed agent $\mathrm{B}$, it indicates ownership attributed to $\mathrm{B}$. Note that the stimulus $\mathrm{s}$ triggering preparation of action a can be of any type; for social scenarios, it can be taken as a body state (e.g., face expression) of the other agent $B$ which is observed. An action effect state $b$ can be any state of the world (possibly including body states).

In accordance with (3) in Section 2, the prior ownership state $\mathrm{PO}(\mathrm{a}, \mathrm{b}, \mathrm{c}, \mathrm{s})$ is affected by the preparation state $\mathrm{PA}(\mathrm{a})$ for the action $a$, the sensory representation $\mathrm{SR}(\mathrm{b})$ of the (predicted) effect $b$, the sensory representation $\mathrm{SR}(\mathrm{s})$ of the stimulus $\mathrm{s}$, and the sensory representation $\mathrm{SR}(\mathrm{c})$ of the context c; see the four arrows to $\mathrm{PO}(\mathrm{a}, \mathrm{b}, \mathrm{c}, \mathrm{s})$ in Figure 2. Similarly, as expressed in (4) in Section 2, the retrospective ownership state $\mathrm{RO}(\mathrm{a}, \mathrm{b}, \mathrm{c}, \mathrm{s})$ is affected by the sensory representation $\mathrm{SR}(\mathrm{c})$ of the context $c$, the sensory representation $S R(b)$ of the effect $b$ of the action, the prior ownership state $\mathrm{PO}(a, b, c, s)$, and the execution $\mathrm{EA}(\mathrm{a})$ of the action a; see the arrows to RO(a, b, c, s) in Figure 2.

Action prediction, expressed in (1) in Section 2, is modelled by the connection from the action preparation $\mathrm{PA}(\mathrm{a})$ to the sensory representation $\mathrm{SR}(\mathrm{b})$ of the effect b. Suppression of the sensory representation of the effect, expressed as (2) in Section 2, is modelled by the (inhibiting) connection from the prior ownership state $\mathrm{PO}(\mathrm{a}, \mathrm{b}, \mathrm{c}, \mathrm{s})$ to sensory representation $\mathrm{SR}(\mathrm{b})$. The control exerted by the prior ownership state, expressed in (5) in Section 2, is modelled by the connection from $P O(a, b, c, s)$ to EA(a). Finally, acknowledging of ownership, expressed in (6) in Section 2, is modelled by the connection from the retrospective ownership state $\mathrm{RO}(\mathrm{a}, \mathrm{b}, \mathrm{c}, \mathrm{s})$ to the communication effector state $\mathrm{EO}(\mathrm{a}, \mathrm{b}, \mathrm{c}, \mathrm{s})$.

\begin{tabular}{|r|l|}
\hline notation & description \\
\hline $\mathrm{WS}(\mathrm{W})$ & world state W (W is a context c, stimulus s, or effect b) \\
\hline $\mathrm{SS}(\mathrm{W})$ & sensor state for W \\
\hline $\mathrm{SR}(\mathrm{W})$ & sensory representation of $\mathrm{W}$ \\
\hline $\mathrm{PA}(\mathrm{a})$ & preparation for action a \\
\hline $\mathrm{EA}(\mathrm{a})$ & execution of action a \\
\hline $\mathrm{PO}(\mathrm{a}, \mathrm{b}, \mathrm{c}, \mathrm{s})$ & prior ownership state for action a with $\mathrm{b}, \mathrm{c}$, and $\mathrm{s}$ \\
\hline $\mathrm{RO}(\mathrm{a}, \mathrm{b}, \mathrm{c}, \mathrm{s})$ & retrospective ownership state for a with $\mathrm{b}, \mathrm{c}$, and $\mathrm{s}$ \\
\hline $\mathrm{EO}(\mathrm{a}, \mathrm{b}, \mathrm{c}, \mathrm{s})$ & communication of ownership of a with $\mathrm{b}, \mathrm{c}$, and $\mathrm{s}$ \\
\hline
\end{tabular}

Table 1 State properties used 
Connections between state properties (the arrows in Figure 2) have weights $\omega_{k}$, as indicated in Table 2. In this table the column LP refers to the (temporally) Local Properties LP1 to LP9 presented below. A weight $\omega_{\mathrm{k}}$ has a value between -1 and 1 and may depend on the specific context c, stimulus s, action a and/or effect state b involved. By varying these connection strengths, different possibilities for the repertoire offered by the model can be realised. Note that usually weights are assumed non-negative, except for the inhibiting connections, such as $\omega_{20}$ which models suppression of the sensory representation of effect $b$.

\begin{tabular}{|c|c|c|c|}
\hline from states & to state & weights & LP \\
\hline $\mathrm{SS}(\mathrm{W})$ & $\mathrm{SR}(\mathrm{W})$ & $\omega_{1}$ & $\mathrm{LP} 1$ \\
\hline $\mathrm{PA}(\mathrm{a}), \mathrm{PO}(\mathrm{a}, \mathrm{b}, \mathrm{self}, \mathrm{s}), \mathrm{SS}(\mathrm{b})$ & $\mathrm{SR}(\mathrm{b})$ & $\omega_{2}, \omega_{20}, \omega_{3}$ & $\mathrm{LP} 2$ \\
\hline $\mathrm{SR}(\mathrm{s}), \mathrm{SR}(\mathrm{b})$ & $\mathrm{PA}(\mathrm{a})$ & $\omega_{4}, \omega_{5}$ & $\mathrm{LP} 3$ \\
\hline $\mathrm{SR}(\mathrm{c}), \mathrm{SR}(\mathrm{s}), \mathrm{SR}(\mathrm{b}), \mathrm{PA}(\mathrm{a})$ & $\mathrm{PO}(\mathrm{a}, \mathrm{b}, \mathrm{c}, \mathrm{s})$ & $\omega_{6}, \omega_{7}, \omega_{8}, \omega_{9}$ & $\mathrm{LP} 4$ \\
\hline $\mathrm{PO}(\mathrm{a}, \mathrm{b}, \mathrm{self}, \mathrm{s}), \mathrm{PA}(\mathrm{a})$ & $\mathrm{EA}(\mathrm{a})$ & $\omega_{10}, \omega_{11}$ & LP5 \\
\hline $\mathrm{EA}(\mathrm{a})$ & $\mathrm{WS}(\mathrm{b})$ & $\omega_{12}$ & LP6 \\
\hline $\mathrm{WS}(\mathrm{W})$ & $\mathrm{SS}(\mathrm{W})$ & $\omega_{13}$ & $\mathrm{LP} 7$ \\
\hline $\mathrm{SR}(\mathrm{c}), \mathrm{SR}(\mathrm{b}), \mathrm{PO}(\mathrm{a}, \mathrm{b}, \mathrm{c}, \mathrm{s}), \mathrm{EA}(\mathrm{a})$ & $\mathrm{RO}(\mathrm{a}, \mathrm{b}, \mathrm{c}, \mathrm{s})$ & $\omega_{14}, \omega_{15}, \omega_{16}, \omega_{17}$ & $\mathrm{LP} 8$ \\
\hline $\mathrm{RO}(\mathrm{a}, \mathrm{b}, \mathrm{c}, \mathrm{s})$ & $\mathrm{EO}(\mathrm{a}, \mathrm{b}, \mathrm{c}, \mathrm{s})$ & $\omega_{18}$ & $\mathrm{LP} 9$ \\
\hline
\end{tabular}

Table 2 Overview of the connections and their weights

Below, the dynamics following the connections between the states in Figure 2 are described in more detail. This is done for each state by a dynamic property specifying how the activation value for this state is updated based on the activation values of the states connected to it (the incoming arrows in Figure 2). The modelling approach is based on continuous-time recurrent neural networks, as proposed by Beer (1995), adopting elements from (Hopfield 1982, 1984). Note that modeling the causal relations discussed in neurological literature and shown in Figure 2 does not follow specific single neurons but abstracts them to cognitive or mental states. By this abstraction neurological knowledge is lifted to a mental (cognitive/affective) modelling level. See also the discussion in (Bickle, 1998) which he illustrates for the higher level (e.g., folk psychological) in relation to the lower-level (e.g., neurobiological) explanation in the context of Hawkin and Kandel's (1984a,1984b) work:

'Of course, the functional profiles assigned to cognitive states on Hawkin and Kandel's neurobiological account are much more fine-grained and detailed, for that account recognizes distinctions and connections that folk psychology either lumps together or leaves extremely vague (...) Here again, however, we can expect that injection of some neurobiological details back into folk psychology would fruitfully enrich the latter, and thus allow development of a more fine-grained folkpsychological account that better matches the detailed functional profiles that neurobiology assigns to its representational states. There is no principled reason against such enrichment.' (Bickle, 1998, pp. 207-208)

According to this approach, during processing each state has an activation level or strength represented by a real number between 0 and 1 ; variables $V$ (possibly with subscripts) run over these values. In dynamic property specifications, this is added as a last argument to the state property expressions (an alternative notation activation $(p, v)$ with $p$ a state property has not been used for the sake of notational simplicity). Activation levels of states affect activation levels of other states (to which they are causally connected; see the arrows in Figure 2) by some state update 
specification; below these are specified as Local Properties (LP) for each of the different states. Such a state update specification uses a parameter $\gamma$ as a speed factor, indicating the speed by which the activation level is updated upon received input from other states. For a state causally depending on multiple other states, values for incoming activation levels are combined, using a combination function $f$ which is a function for which different choices can be made. For example, the identity function $f(W)=W$ can be chosen or a combination function based on some threshold function $t h$ :

$$
f\left(X_{1}, \ldots, X_{k}\right)=\operatorname{th}\left(X_{1}+\ldots+X_{k}\right)
$$

This threshold function th can have different forms, for example a discontinuous one:

$$
\begin{aligned}
\operatorname{th}(W)=0 & \text { if } \quad W<\tau \\
1 & \text { if } \quad W \geq \tau
\end{aligned}
$$

for some threshold value $\tau$. Another option is the continuous logistic threshold function $\operatorname{th}(\sigma, \tau, X)$ of the form

$$
\operatorname{th}(\sigma, \tau, W)=\left(\left(1 /\left(1+e^{-\sigma(W-\tau)}\right)\right)-\left(1 /\left(1+e^{\sigma \tau}\right)\right)\right)\left(1+e^{-\sigma \tau}\right)
$$

with $\sigma$ a steepness and $\tau$ a threshold value. Note that for higher values of $\sigma \tau$ (e.g., $\sigma$ higher than

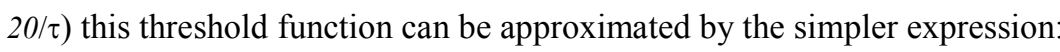

$$
\operatorname{th}(\sigma, \tau, W)=1 /\left(1+e^{-\sigma(W-\tau)}\right)
$$

In the example simulations, for the states that are affected by only one state (i.e., in LP1, LP6, LP7, LP9), $f$ is taken the identity function $f(W)=W$, and for the other states $f$ is a combination function based on the logistic threshold function $\operatorname{th}(\sigma, \tau, W)$. In this choice common practice is followed, but other types of combination functions might be used as well.

The computational agent model has been formalised according to this approach in two different ways so that readers who are familiar with one of these two formats can easily access it: (1) using a differential equations format (see Box 1), and (2) using the hybrid LEADSTO format; cf. [Bosse et al., 2007] (see Box 2). Within LEADSTO a dynamic property or temporal causal relation $\mathrm{a} \rightarrow \mathrm{b}$ denotes that when a state property a (or conjunction thereof) occurs, then after a certain time delay, state property $\mathrm{b}$ will occur. Below, this delay will be taken as a uniform time step $\Delta \mathrm{t}$.

In the text below, for each of these state update specifications a semiformal description is given, whereas a formal specification in differential equation format (one differential equation per Local Property LP) is shown in Box 1 and a formal specification in the hybrid LEADSTO format is shown in Box 2. Note that to make a graphical distinction, in the LEADSTO specification capitals are used (as in the tables and in Figure 2), whereas in the differential equations no capitals are used. The first property LP1 describes how sensory representations are generated for context $\mathrm{c}$ and stimulus $\mathrm{s}$, and effect state $\mathrm{b}$ (together indicated by variable $\mathrm{W}$ ).

\section{LP1 Sensory representation for a sensor state}

If the sensor state for $\mathrm{W}$ has level $V_{l}$

and the sensory representation of $\mathrm{W}$ has level $V_{2}$

then after duration $\Delta t$ the sensory representation of $\mathrm{W}$ will have level $V_{2}+\gamma\left[f\left(\omega_{1} V_{l}\right)-V_{2}\right] \Delta \mathrm{t}$.

The sensory representation of an effect state $b$ as described by property LP2 is not only affected by a corresponding sensor state for $\mathrm{b}$ (which in turn is affected by the world state), as in LP1, but also by two action-related states: 
- via the predictive loop by a preparation state, to predict the effect b of a prepared action a (see (1) in Section 2)

- by an inhibiting connection from the prior self-ownership state, to suppress the sensory representation of the effect $b$ of the action a, once it is initiated (see (2) in Section 2)

This is expressed in dynamic property LP2. Note that for this suppressing effect the connection weight $\omega_{20}$ from prior ownership state for action a to sensory representation for effect $\mathrm{b}$ is taken negative, for example $\omega_{20}=-1$.

\section{LP2 Sensory representation for an effect state}

If the preparation state for action a has level $V_{l}$

and the prior self-ownership of action a for $\mathrm{b}$, self, and $\mathrm{s}$ has level $V_{2}$

and the sensor state for state $\mathrm{b}$ has level $V_{3}$

and the sensory representation of state $\mathrm{b}$ has level $V_{4}$

then after duration $\Delta t$ the sensory representation of state b will have level $V_{4}+\gamma\left[f\left(\omega_{2} V_{1}, \omega_{20} V_{2}, \omega_{3} V_{3}\right)-V_{4}\right] \Delta t$

Preparation for action a is affected by a sensory representation of stimulus s (triggering the action), and also strengthened by predicted effect $b$ of the action:

\section{LP3 Preparing for an action}

If sensory representation of s has level $V_{l}$ and sensory representation of $\mathrm{b}$ has level $V_{2}$ and the preparation for action a has level $V_{3}$ then after duration $\Delta t$ the preparation state for action a will have level $V_{3}+\gamma\left[f\left(\omega_{4} V_{1}, \omega_{5} V_{2}\right)-V_{3}\right] \Delta t$.

Prior ownership of an action a is generated by LP4 (see (3) in Section 2).

\section{LP4 Generating a prior ownership state}

If the sensory representation of context c has level $V_{l}$ and the sensory representation of s has level $V_{2}$

and sensory representation of $\mathrm{b}$ has level $V_{3}$

and the preparation for action a has level $V_{4}$

and prior ownership of a for b, c, and s has level $V_{5}$

then after $\Delta t$ prior ownership of a for c, s, and b will have level $V_{5}+\gamma\left[f\left(\omega_{6} V_{l}, \omega_{7} V_{2}, \omega_{8} V_{3}, \omega_{9} V_{4}\right)-V_{5}\right] \Delta t$.

In case the context $c$ is self, the prior ownership state strengthens the initiative to perform a as a self-generated action: executing a prepared action depends on whether a prior self-ownership state (for the agent self) is available for this action (see (5) in Section 2). This models control over the actual execution of the action (go/no-go decision) and can, for example, be used to veto the action in a late stage of preparation. This is modelled by LP5.

\section{LP5 Action execution}

If prior ownership of a for $\mathrm{b}$, self, and s has level $V_{l}$

and preparation for action a has level $V_{2}$

and the action execution state for a has level $V_{3}$

then after duration $\Delta t$ the action execution state for a will have level $V_{3}+\gamma\left[f\left(\omega_{10} V_{l}, \omega_{11} V_{2}\right)-V_{3}\right] \Delta t$.

Property LP6 describes in a straightforward manner how execution of action a affects the world state b.

\section{LP6 From action execution to effect state}

If the execution state for action a has level $V_{l}$, and world state $\mathrm{b}$ has level $V_{2}$

then after $\Delta t$ world state b will have level $V_{2}+\gamma\left[f\left(\omega_{12} V_{1}\right)-V_{2}\right] \Delta t$. 
The following property models how sensor states are updated. It applies to stimulus s, effect b, and context c (indicated by variable $\mathrm{W}$ ).

\section{LP7 Generating a sensor state for a world state}

If $\quad$ world state $\mathrm{W}$ has level $V_{l}$

and the sensor state for $\mathrm{W}$ has level $V_{2}$

then after $\Delta t$ the sensor state for $\mathrm{W}$ will have level $V_{2}+\gamma\left[f\left(\omega_{13} V_{l}\right)-V_{2}\right] \Delta t$.

A retrospective ownership state takes into account the prior ownership, the execution of the action, the context, and the sensory representation of the action's effect (see (4) in Section 2):

\section{LP8 Generating a retrospective ownership state}

If the sensory representation of context c has level $V_{l}$, and the sensory representation of effect state $\mathrm{b}$ has level $V_{2}$ and prior ownership of a for b, c, and s has level $V_{3}$ and the execution state for action a has level $V_{4}$ and retrospective ownership of a for b, c, and s has level $V_{5}$ then after $\Delta t$ retrospective ownership of a for $\mathrm{b}, \mathrm{c}$, and s will have level $V_{5}+\gamma\left[f\left(\omega_{14} V_{l}, \omega_{15} V_{2}, \omega_{16} V_{3}, \omega_{17} V_{4}\right)-V_{5}\right] \Delta t$.

Note that LP8 applies for context self as context, but also to an observed other agent B. For an observed other agent as context the connection strength $\omega_{17}$ in LP8 is assumed 0 or negative; in the simulated scenarios discussed in Section 3 it was taken $\omega_{17}=-1$. The communication to attribute authorship (to any context c) depends on the retrospective ownership state as specified in LP9 (see (6) in Section 2).

\section{LP9 Communication of ownership awareness}

If retrospective ownership of a for b, c, and s has level $V_{l}$, and communication of a for $\mathrm{b}, \mathrm{c}$, and $\mathrm{s}$ has level $V_{2}$

then after duration $\Delta t$ communication of a for b, c, and s will have level $V_{2}+\gamma\left[f\left(\omega_{18} V_{l}\right)-V_{2}\right] \Delta t$.

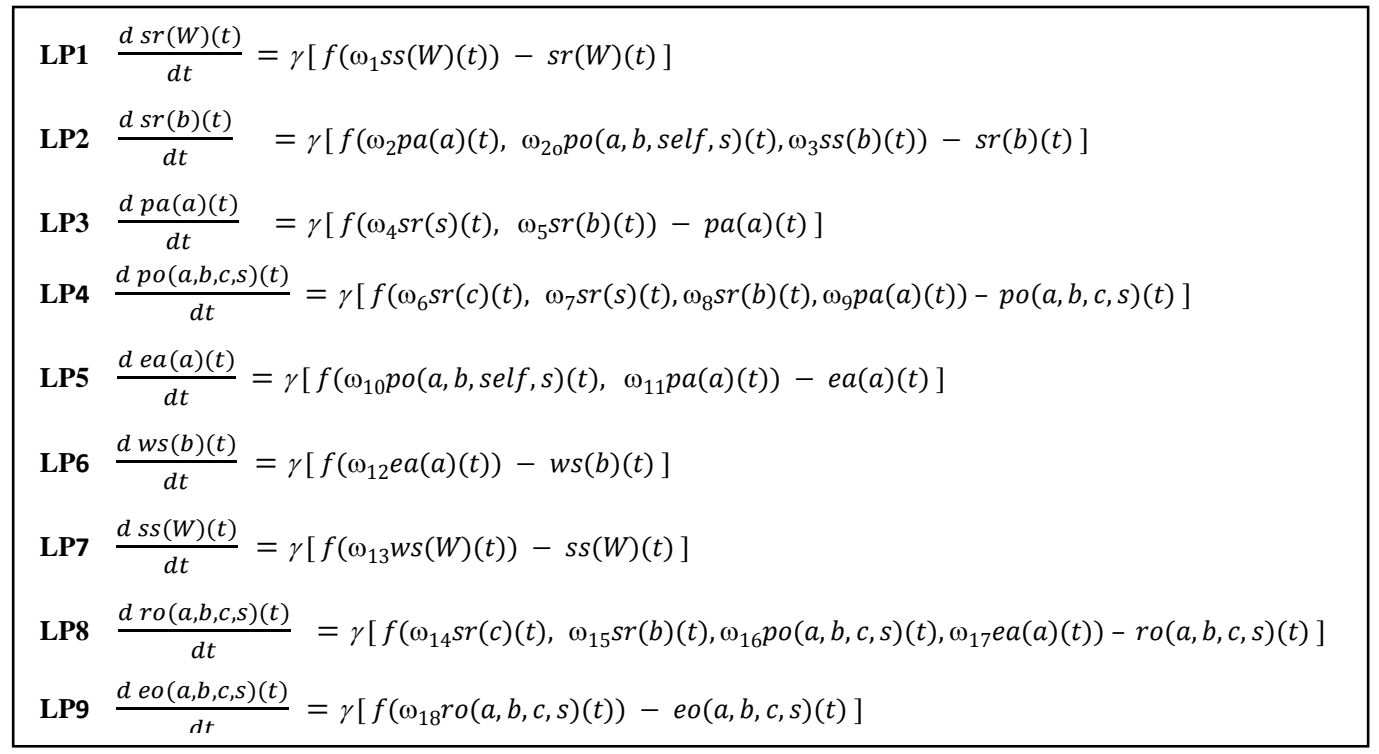

Box 1 Formal specification of the model in differential equation format 


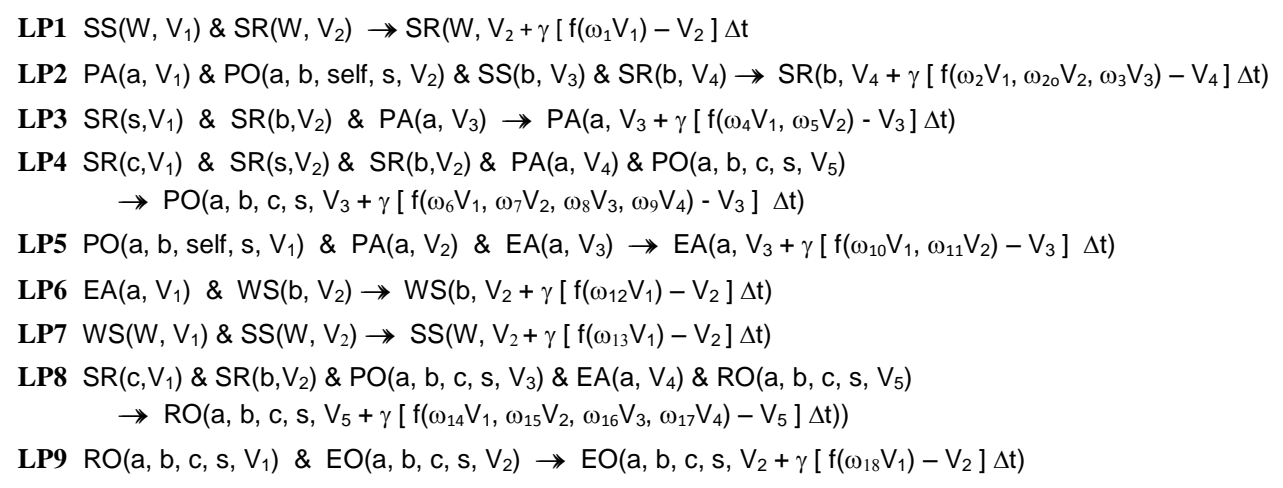

Box 2 Formal specification of the model in LEADSTO format

\section{Simulation of Example Scenarios}

In this section simulations are discussed for a number of example scenarios, which all involve the occurrence of a preparation state for an action a, triggered by some stimulus s. These scenarios relate to phenomena in the literature, as discussed in Section 1. They have been generated based on the specification in differential equation format shown in Box 1. Alternatively, the LEADSTO software environment could be (but in this case actually has not been) used; both is equally well possible. First a scenario is addressed where the prepared action has satisfactory predicted effects and therefore is executed; in this case both prior and retrospective self-ownership states occur. Next, a case is considered where the prepared action lacks positive predicted effects, and is therefore not executed: a no-go decision, or vetoing. Only a rather low prior self-ownership state is developed and no retrospective self-ownership state. In the third case, a poor action prediction capability is modelled, which leads to a not very high prior self-ownership state, but sufficient to actually execute the prepared action. In this case no retrospective self-ownership state occurs, as the sensory representation of the effect stays low. In the fourth case, the stimulus triggering the action preparation is the observation of another agent performing the action. In this case a low prior self-ownership state is generated, but high prior and retrospective other-ownership states. This models mirroring of and attribution to the other agent. Note that the parameter values for the connection strengths used for these different scenarios are not unique. Similar patterns are obtained when they are in a certain range.

\subsection{Normal Execution and Attribution of an Action}

The first case considered describes a situation where the context $\mathrm{c}$ is the agent itself, and a stimulus $s$ occurs. The action effect $b$ is considered positive for the agent. To make things more specific, imagine the following:

- context $\mathrm{c}$ is that you are working on your computer

- $\quad$ stimulus $s$ is that you need a certain program $P$

- action a is clicking on a specific icon I on the desktop of your computer

- effect $b$ of action a is that program $\mathrm{P}$ is opened.

The first scenario is as follows: 


\section{Scenario 1}

- external stimulus s occurs and triggers preparation of action a (to click on icon I)

- based on the preparation state for a the sensory representation of predicted effect $b$ (that program $\mathrm{P}$ is opened) of a is generated

- based on this positive predicted effect and the other states a prior self-ownership state for action a (to click on icon I) is generated

- this prior self-ownership state for action a leads to actual execution of action a (mouseclick on I)

- the execution of a affects $b$ in a positive manner (program P opens) and, via sensing, also the sensory representation of $\mathrm{b}$ (that program $\mathrm{P}$ is opened)

- at the same time the sensory representation of $b$ is suppressed due to the prior self-ownership state

- based on the generated states, after the execution of action a the agent develops a retrospective selfownership state (for opening program P by clicking on icon I)

- finally the agent communicates this self-ownership ('I opened program P by clicking on icon I')

The simulation of this scenario is shown in Figure 3. Parameter values used (in all scenarios set by hand) can be found in Table 3. The step size taken is $\Delta t=0.25$. All relevant connection strengths not mentioned in this table were taken 1 . The slow value 0.3 for $\gamma$ was applied for external processes (action execution, effect generation and effect sensing) modelled by LP5, LP6, and LP7, and the fast value 0.6 for $\gamma$ for the internal processes modelled by the other LP's.

In Figure 3 it is shown that (after sensing the stimulus), the preparation for action a starts around time point 2 , and the representation of the predicted effect $b$ around time point 3 . As a result of this, around time point 6 the prior self-ownership state starts to develop, which leads to the execution of the action, starting around time point 7. In the meantime the representation of the action effect b is suppressed (cf. [Blakemore et al., 1999; Blakemore et al., 2000; Fourneret et al., 2002]), causing a dip in the graph around time point 10 . When the execution of the action a is taking place, the sensing of its effect $b$ in the world has a positive impact on the representation of $b$ from time point 10 on, and the retrospective self-ownership state is developed, starting from around time 15. After this, the communication of the self-ownership takes place from time point 20 .

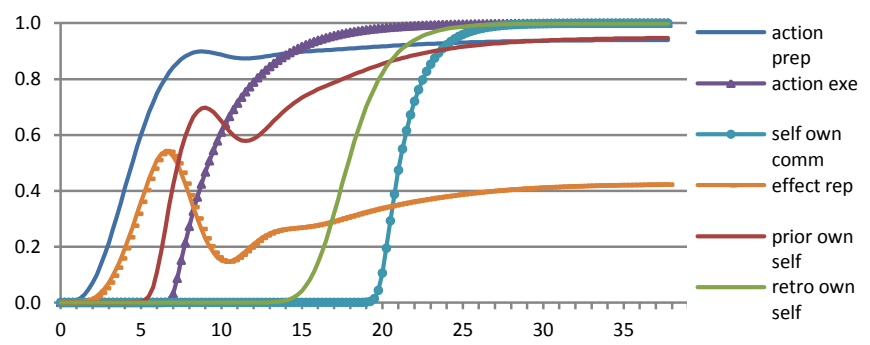

Figure 3 Executing an action with ownership states (scenario 1)

Note that in this case both the prior and the retrospective self-ownership state reach levels close to 1 (prior self-ownership approaching 0.95, and retrospective self-ownership approaching 1). Moreover, note that when the stimulus is taken away, all activation levels will go down to 0 , and will come up again when the stimulus reoccurs. 


\begin{tabular}{|r|c||r|c|c|}
\hline \multicolumn{2}{|c|}{ connections } & threshold and steepness for state & $\boldsymbol{\tau}$ & $\boldsymbol{\sigma}$ \\
\hline$\omega_{3}$ & 0.5 & action preparation & 0.4 & 4 \\
\hline$\omega_{20}$ & -1 & effect representation & 0.2 & 4 \\
\hline$\omega_{2}$ & 0.8 & action execution & 1.2 & 20 \\
\hline$\omega_{4}$ & 0.8 & prior self-ownership & 3 & 8 \\
\hline$\omega_{5}$ & 0.8 & retrospective self-ownership & 3 & 20 \\
\hline$\gamma$ & $0.6 / 0.3$ & self-ownership communication & 0.8 & 40 \\
\hline
\end{tabular}

Table 3: Parameter values for the first scenario

\subsection{Vetoing a Prepared Action Due to Unsatisfactory Predicted Effect}

The second case considered describes a situation similar to the previous one (the context $\mathrm{c}$ is the agent itself, and a stimulus s occurs), but where the action a triggered by stimulus s has an effect b' which is not particularly positive for the agent; here a hardly has an impact on effect $b$ which would have been positive. Prediction capabilities are assumed correct in this case, so no high level of $b$ is correctly predicted for a. For the imagined program opening context this means that it is predicted that clicking on icon I does not open program P but a different program P'. Nevertheless it is assumed that the stumulkus triggers preparation for clicking on icon I (maybe because in the past on the same position the icon for prgram $\mathrm{P}$ was placed). For this situation the following variation on the previous scenario is considered:

\section{Scenario 2}

- external stimulus s occurs and triggers preparation of action a (to click on icon I)

- based on the preparation state of a only a low level for the sensory representation of predicted effect $b$ of a is generated (no prediction that program $\mathrm{P}$ will be opened but that different program $\mathrm{P}^{\prime}$ will be opened)

- based on this low predicted effect $b$ and the other states a low level of a prior self-ownership state for action a is generated (low prior ownership to click on icon I)

- the low prior self-ownership state for a does not lead to actual execution of action a; the action a can be considered vetoed (no click on icon I)

- the agent develops no retrospective self-ownership state for a (no retrospective self-ownership for clicking on icon I)

- the agent does not communicate self-ownership for a

The simulation of this scenario is shown in Figure 4. This scenario was modelled by taking the connection strength for the prediction of effect $b$ for action a low: $\omega_{2}=0.3$ instead of 0.8 . Values for the other parameters were the same as in Table 3 .

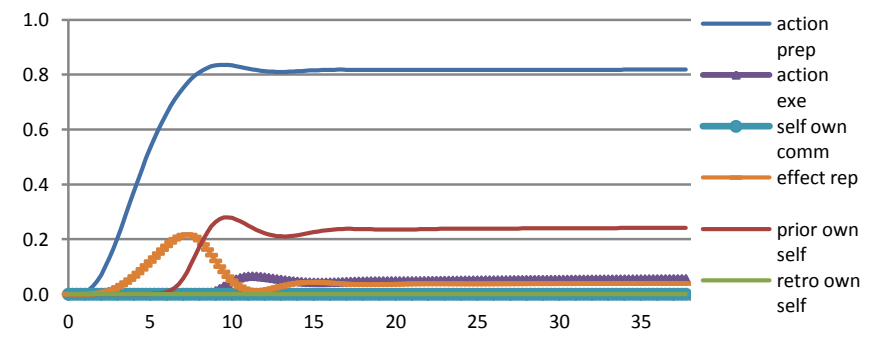

Figure 4 Vetoing an action with no positive prediction (scenario 2)

In Figure 4 it is shown that (after sensing the stimulus), again the preparation for action a starts around time point 2 , and the representation of the predicted effect $b$ around time point 3 . However, 
the predicted effect is much lower compared to the previous scenario. As a result of this low prediction, the prior self-ownership state starting to develop around time point 6 , also stays at a low level. Therefore the execution of the action also stays very low. Due to these circumstances, no retrospective self-ownership state and no communication of self-ownership occur.

\subsection{Effects of Poor Prediction; Schizophrenia Case}

The third case considered describes a situation where again the context $c$ is the agent itself, and stimulus s occurs. The action effect for action a is $b$, which in principle is positive for the agent, like in the first situation above. However, due to poor prediction capabilities this effect is not (fully) internally predicted. This is what is assumed to happen in patients with schizophrenia, as discussed, for example, in (Synofzik et al., 2010; Voss et al., 2010). For this situation the following scenario is considered:

\section{Scenario 3}

- $\quad$ stimulus s occurs and triggers preparation of action a (to click on icon I)

- based on the preparation state for a only a relatively low level of the sensory representation of the predicted effect $b$ of $a$ is generated, due to poor prediction capabilities (low prediction that program $\mathrm{P}$ will be opened)

- based on this relatively low predicted effect and the other states a relatively low level of a prior selfownership state for action a is generated (low prior self-ownership for clicking on icon I)

- this prior self-ownership state level for action a is still sufficient to lead to actual execution of action a (mouseclick on icon I)

- the execution of a affects b (program $\mathrm{P}$ is opened) in a positive manner and (via sensing) the sensory representation of $b$

- the sensory representation of $b$ (that program $\mathrm{P}$ is opened) is suppressed to a certain extent due to the (relatively low) prior self-ownership state

- due to the relatively low level for the sensory representation of effect $b$ (and prior self-ownership state) the agent develops no retrospective self-ownership state for action a (no retrospective self-ownership for clicking on icon I)

- the agent does not communicate self-ownership for action a

The simulation of this scenario is shown in Figure 5. This scenario was modelled by taking the connection strength for the prediction of effect b for action a moderately low: $\omega_{2}=0.4$. Values for the other parameters were again the same as in Table 3. For this case $\Delta t=0.1$ was taken instead of 0.25 , and the simulation was shown up to time point 75 .

In Figure 5 it is shown that as in the previous scenarios, the preparation for action a starts around time point 2 , and the representation of the predicted effect $b$ around time point 3 . The predicted effect is substantially lower compared to the first scenario, but higher than in the second scenario. As a result of this moderately low prediction, the prior self-ownership state, starting to develop around time point 6, also stays at a moderate level (first around 0.4, later going up to almost 0.7 ); this is substantially higher than in the second scenario where the lower level led to a veto for the action. Therefore, in contrast to the previous scenario, this level turns out high enough for the execution of the action starting around time point 9 . Nevertheless, only a low level of the retrospective self-ownership state is developed (becoming approximately 0.15), and no communication of self-ownership takes place. 

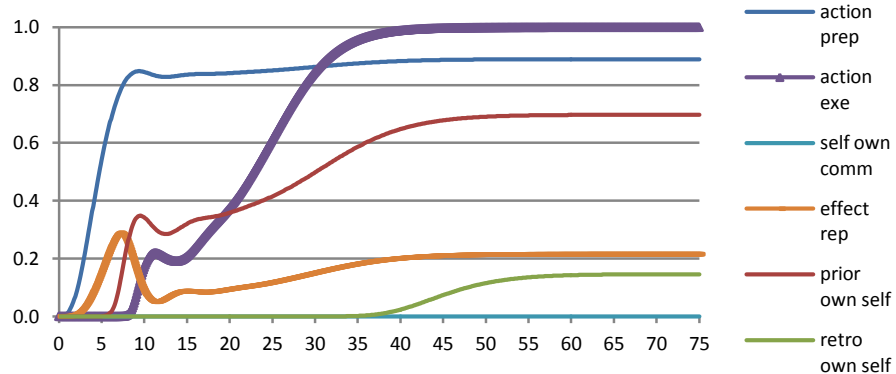

Figure 5 Poor prediction implies no retrospective self-ownership (scenario 3)

\subsection{Satisfactory Predicted Effects but Unsatisfactory Actual Effects}

The fourth case considered describes a situation where again the context $c$ is the agent itself, and stimulus s occurs. The predicted action effect for action a is $\mathrm{b}$, which in principle is positive for the agent, like in the first situation above. However, after executing the action, it turns out that $\mathrm{b}$ is not an actual effect of the action. This is another way of modelling a mismatch between prediction and actual outcome. For this situation the following scenario is considered:

\section{Scenario 3}

- external stimulus s occurs and triggers preparation of action a (to click on icon I)

- based on the preparation state for a the sensory representation of predicted effect $b$ of a is generated (that program $\mathrm{P}$ will be opened)

- based on this positive predicted effect and the other states a prior self-ownership state for action a is generated (to click on icon I)

- this prior self-ownership state for action a leads to actual execution of action a (mouseclick on icon I)

- the execution of a does not affect $b$ in a positive manner (program $\mathrm{P}$ is not opening, but a different program $\mathrm{P}^{\prime}$ ) and; therefore $\mathrm{b}$ is not sensed, and no contribution occurs to the sensory representation of $\mathrm{b}$

- at the same time the sensory representation of $b$ is suppressed due to the prior self-ownership state

- based on the generated states, the agent develops no retrospective self-ownership state (no retrospective self-ownership for clicking on icon I)

- the agent does not communicate self-ownership for action

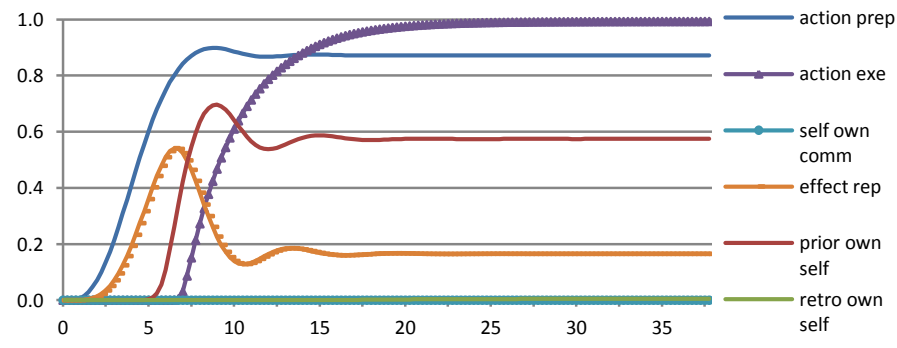

Figure 6 Deviation of actual effect from predicted effect implies no retrospective self-ownership (scenario 4)

The simulation of this scenario is shown in Figure 6. This scenario was modelled by taking the connection strength for the actual effect $b$ for action a very low: $\omega_{12}=0$. Values for the other parameters were again the same as in Table 3. For this case $\Delta t=0.25$ was taken. 
In Figure 6 it is shown that as in the previous scenarios, the preparation for action a starts around time point 2, and the representation of the predicted effect $b$ around time point 3 . The predicted effect is comparable to the first scenario. As a result of this prediction, the prior selfownership state, develops from around time point 6 on going up to 0.7 ). Therefore, this level is high enough for the execution of the action starting around time point 9. However, the expected effect $b$ of the action does not actually occur. Moreover due to the prior ownership state the sensory representation of the effect is suppressed. Therefore immediately after time point 6 , the sensory representation of the effect goes down. As a consequence no retrospective self-ownership state is developed, and no communication of self-ownership takes place.

\subsection{Mirroring Another Agent}

In contrast to the first four scenarios, the fifth case describes a situation where the context $c$ is another agent, and the stimulus $\mathrm{s}$ is the observation of the other agent performing action a (agent $\mathrm{B}$ is clicking on icon I). The action effect for action $\mathrm{a}$ is $\mathrm{b}$ (program $\mathrm{P}$ is opened) and is predicted in a correct manner, as in the first scenario. The scenario for this fourth case is as follows:

\section{Scenario 5}

- external stimulus s which is an observed action a performed by another agent (by mirroring) triggers preparing action a (to click on icon I)

- based on the preparation state for a the sensory representation of the predicted effect $b$ of a is generated (that program $\mathrm{P}$ will be opened)

- based on this predicted effect and the other states (among which the other agent as context) a high level of a prior other-ownership state for action a is generated, and a low level of a prior self-ownership state (other-ownership for clicking on icon I; no self-ownership for clicking on icon I)

- the low prior self-ownership state for action a leads to no actual execution of action a (vetoing; no own mouseclick on icon I))

- as the prior self-ownership state has a low level, not much suppression of the representation of effect b takes place

- based on the generated states, the agent develops a retrospective other-ownership state, and no retrospective self-ownership state (retrospective other-ownership for agent B opening program $\mathrm{P}$ by clicking on icon I)

- finally the agent communicates this other-ownership a ('you opened program P by clicking on icon I')

The simulation of this scenario is shown in Figure 7. This scenario was modelled by taking the connection strength $\omega_{4}$ for mirroring from the specific stimulus representation (observed action) to preparation state 0.5 . The connection strength $\omega_{2}$ for the prediction of effect $\mathrm{b}$ for action a is 0.8 , as in the first scenario. The threshold and steepness values for prior and retrospective otherownership states were taken 3 and 8 , resp. 2.4 and 20. For this case $\Delta t=0.25$ was taken. Values for the other parameters were the same as for the first scenario.

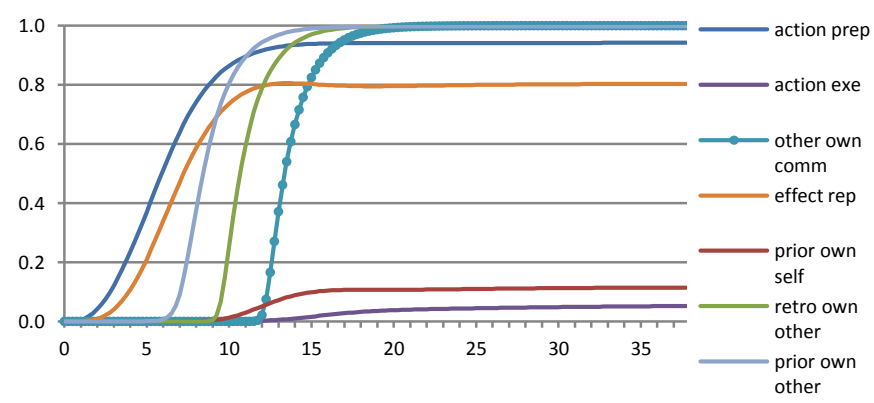

Figure 7 Mirroring another agent (scenario 5) 
In Figure 7 it is shown that after sensing the observed action, as in the first scenario the preparation for action a starts around time point 2 , and the representation of the predicted effect $b$ around time point 3. As a result of this, around time point 6 the prior other-ownership state starts to develop, whereas the prior self-ownership state stays very low. Therefore execution of a is suppressed. After time point 9 also the retrospective other-ownership state is generated, which leads to communication of other-ownership after time point 12. All other states stay low.

\section{Relations to Neurological Findings}

In this section the question is addressed how the states and dynamical relations of the model described in Section 3 relate to neurological states and mechanisms. In particular, this concerns the internal states: sensory representations, preparations, and the prior and retrospective ownership states (in Figure 2 the states in the box). First of all, for the sensory representations of stimulus and context and action effect, these are considered to be related to sensory neurological states. Secondly, the preparation state used is assumed to have a mirroring function as well, so this state may be related to (a group of) mirror neurons (in the classical sense); (e.g., Iacoboni, 2008a).

The remaining two types of states, the prior and retrospective ownership states are the most crucial states in the presented model. A prior ownership state has control over the execution of a (prepared) action as one of its most important functions. This type of state has roughly the following behaviour in relation to execution:

- it is active if a prepared action is (to be) executed

- it is not active when no execution (is to) take(s) place

Interestingly, in recent human single cell recording experiments, specific neurons have been found with activation patterns that have some correlation to execution of an action, in particular, in work reported in (Mukamel et al., 2010; Fried, Mukamel, Kreiman, 2011); see also (Keysers and Gazzola, 2010; Iacoboni, 2008a; Iacoboni, 2008b; Iacoboni and Dapretto, 2006). For example, Iacoboni (2008b) describes these experiments in patients with epilepsy undergoing pre-surgical evaluation of the foci of epilepsy as follows; see also (Iacoboni, 2008a, pp. 201-203).

'From a total of 14 patients, we have recorded the activity of approximately 500 neurons located in three sectors of the mesial frontal cortex: the ventral and dorsal sectors of the anterior cingulate cortex and the pre-supplementary motor cortex (SMA)/SMA proper complex.' (Iacoboni, 2008b, p. 30)

Some of the main findings are that neurons with mirror neuron properties were found in all sites in the mesial frontal cortex were recording took place (approximately $12 \%$ of all recorded neurons); half of them related to hand-grasping, and the other half to emotional face expressions. For the relation of their activity to execution the following was found:

'One-third of mirror neurons had excitatory responses during both action execution and action observation. This is the most typical pattern of firing-rate changes observed in monkeys. Onethird of mirror neurons, however, had inhibitory responses during both action execution and action observation. This pattern has also been occasionally observed in monkeys, but much less frequently. The remaining third of mirror neurons in the human frontal cortex had a pattern of firing-rate changes that has never been observed in monkeys, at least not so far.' (Iacoboni, 2008b, p. 30) 
It is in this group of mirror neurons that neurons can be found that show behaviour that is similar to the behaviour of the prior ownership state in the presented model as indicated above. A substantial subset of these latter set of neurons...:

'...have excitatory responses during action execution and inhibitory responses during action observation. Few of these neurons have the opposite pattern, with decreased firing rate during execution and increased firing rate during observation.' (Iacoboni, 2008b, p. 30)

In (Iacoboni, 2008a, 2008b; Iacoboni and Dapretto, 2006) such types of neurons are termed super mirror neurons, to indicate the control function they may have with respect to the execution of an action. Given the similarity in behaviour, it is this type of neurons that can be considered a suitable candidate as a neurological counterpart of prior ownership states. One aspect to be addressed is the timing aspect: are these super mirror neurons active before the execution, or during or after? In the latter case, they could be considered more suitable as candidates for retrospective ownership states. To obtain more evidence, it would be interesting to find out more about temporal dependencies between the respective neural and execution states, for example, using methods as described in (Schippers and Keysers, 2011).

For the remaining type of states to be considered, the retrospective ownership states, a more complex picture occurs. Retrospective ownership states have a strong relation to self-monitoring, as also emphasized in (Arbib and Mundhenk, 2005; Arbib, 2007; Moore and Taggard, 2008). For example, in (David et al., 2008), a number of cortical and subcortical elements are indicated that may relate as neural correlates to retrospective ownership states:

'...several brain areas have been implicated in the sense of agency (...). These include brain regions known to be involved in the motor system such as the ventral premotor cortex (vPMC), the supplementary motor area (SMA and pre-SMA) and the cerebellum as well as regions such as the dorsolateral prefrontal cortex (DLPFC), the posterior parietal cortex (PPC), the posterior segment of the superior temporal sulcus (pSTS) and the insula (...). Unfortunately, the current literature does not yet provide a consistent or clear picture with respect to the exact functions and contributions of these brain regions to the sense of agency.' (David et al. 2008, p. 529)

In (David et al., 2008) a distinction is made into what are called executive functions and supervisory functions, and empirical results in the literature are discussed accordingly.

'In a classificatory attempt, the first group of brain regions (e.g., vPMC, SMA, cerebellum) constitutes a network of sensorimotor transformations and motor control, whereas the second group of brain regions rather represents a set of heteromodal association cortices implicated in various cognitive functions. (...) Accordingly, motor system-related regions may subserve 'executive' functions whereas heteromodal associative regions subserve 'supervisory' functions. However, the proposed classificatory, functional distinction remains speculative requiring further empirical validations. In the following, we summarize the available evidence on the neuroscience of agency in an attempt to putting the empirical results into perspective. The presence of different neural correlates might reflect different agency indicators, subprocesses or levels of agency processing.' (David et al. 2008, pp. 529-530)

So, the possibility is left open that in the end different agency indicators may be distinguished. One of such distinctions may be between unconscious and conscious ownership states; see, for example, (Jeannerod, 2009; Schutz-Bosbach et al., 2009). 


\section{Discussion}

The computational agent model presented in this paper incorporates mechanisms for prior and retrospective ownership states, based on principles from recent neurological theories, in particular from (Moore and Haggard, 2008; Synofzik et al., 2010; Voss et al., 2010). In the model a prior ownership state is affected by prediction of the effects of the action. Actual execution of the action and sensing of its effects can lead to a retrospective ownership state, in particular, when the sensed effects co-occur with the predicted effects. As a prior ownership state may lead to actual execution of the action, it plays an important role as control of the execution of prepared actions. A retrospective ownership state is the basis for acknowledging authorship of an action, for example, in social context, or in a self-reflection context.

In simulated scenarios it was shown how a number of known phenomena can occur. For example, scenarios were shown for vetoing a prepared action due to unsatisfactory predicted effects, and for mirroring an observed action performed by another agent, without imitating the action. Moreover, it was shown how poor action effect prediction capabilities can lead to reduced retrospective ownership states (as, for example, is shown in persons suffering from schizophrenia), and may easily lead to attribution of the self-generated action to another real or imaginary person.

Modeling causal relations discussed in neurological literature in the manner as presented here does not take specific neurons into consideration but uses more abstract cognitive or mental states. In this way abstraction takes place by lifting neurological knowledge to a mental (cognitive/affective) modelling level. The type of computational model that results shows some technical elements from the neural modelling area. More specifically, it takes states as having a certain activation level (as opposed to binary states), thus making reciprocal loops possible. To achieve this, the modelling approach exploits techniques used in continuous-time recurrent neural networks, in line with what is proposed by Beer (1995), adopting elements from (Hopfield 1982, 1984). In particular, for a state causally depending on multiple other states, values for incoming activation levels are combined, using a combination function.

The ownership states are internal cognitive states that are not representations of currently present external things in the way that, for example, sensory representation or belief states are. If the question is posed whether they do represent anything, and if so, what, maybe they can be interpreted as temporal second-order representations or representations of the agent's own internal processes, or representations of past or future behaviour, or a combination of these. This representation question is not the focus of this paper. It may be an interesting topic for further work, for example, in the line of (Bosse, Jonker, and Treur, 2009).

The model distinguishes itself from existing approaches such as in (Wolpert, 1997; Frith, 1992; Frith et al., 2000), among others in that (1) instead of comparison of predicted and sensed effects, the predicted and sensed effects are integrated and provide a kind of combined level, as also indicated in, for example (Moore and Haggard, 2008; Synofzik et al., 2010; Voss et al., 2010), (2) following (Moore and Haggard, 2008) a distinction was made between prior and retrospective ownership states, and (3) both self-ownership and other-ownership are covered. These are also differences with approaches, such as for example (Hindriks et al., 2011), which do not take the neurological angle as a point of departure, as in the current paper.

The obtained computational model can be used as a basis for simulation-based training or in gaming or virtual stories. For the first type of application the idea is to develop a virtual patient based on the model so that, for example, a psychiatrist or psycho-therapist (e.g., during his or her education) can gain insight in the processes in certain types of patients, or it can be used by a therapist to analyse how a certain form of therapy can have its effect on these processes. For the second type of application the idea is to design a system for agent-based virtual stories in which, 
for example, persons with deviations in ownership states play a role (e.g., persons suffering from schizophrenia, and due to that attribute their own actions to other real or imaginary persons), which can be based on the presented model.

Note that the current paper only addresses the occurrence of ownership states. It does address how weak self-ownership can occur but does not address how in some cases imaginary persons can be created to whom actions are attributed; see for example (Collerton et al., 2005; Samsonovich, 2005). In (Treur and Umair, 2011) it is shown how so-called inverse mirroring enables a person to attribute an action to an imaginary person.

\section{References}

Arbib, M.A. (2007). Other faces in the mirror: a perspective on schizophrenia. World Psychiatry, $6,75-78$.

Arbib, M.A., Mundhenk, T.N. (2005). Schizophrenia and the mirror system: an essay. Neuropsychologia, 43, 2005, 268-80.

Beer, R.D. (1995). On the dynamics of small continuous-time recurrent neural networks, Adaptive Behavior, 3, 469-509.

Bickle, J. (1998). Psychoneural Reduction: The New Wave. Cambridge, Mass: MIT Press.

Blakemore, S.-J., Frith, C.D., (2003). Self-awareness and action. Current Opinion in Neurobiology, 13, 219-224

Blakemore, S.-J., Frith, C.D., and Wolpert, D.M. (1999). Spatio-Temporal Prediction Modulates the Perception of Self-Produced Stimuli. J. of Cognitive Neuroscience, 11, 551-559.

Blakemore, S.-J., Smith, J., Steel, R. et al. (2000a). The perception of self-produced sensory stimuli in patients with auditory hallucinations and passivity experiences: evidence for a breakdown in self-monitoring. Psychol. Med., 30, 1131-1139.

Blakemore, S.-J., Wolpert, D.M., and Frith, C.D. (2000b). Why can't you tickle yourself? Neuroreport, 11, 11-16.

Blakemore, S.-J., Wolpert, D.M., and Frith, C.D. (2002). Abnormalities in the awareness of action. Trends in Cognitive Sciences, 6, 237-242.

Bosse, T., Jonker, C.M., Meij, L. van der, and Treur, J. (2007). A Language and Environment for Analysis of Dynamics by Simulation. Intern. J. of Artificial Intelligence Tools, 16, 435-464.

Bosse, T., Jonker, C.M., and Treur, J., (2009). Representation for Reciprocal Agent-Environment Interaction. Cognitive Systems Research Journal, vol. 10, 2009, pp. 366-376.

Claxton, G. (1975). Why can't we tickle ourselves? Perceptual and Motor Skills, 41, 335-338.

Collerton, D., Perry, E., McKeith, I. (2005). Why people see things that are not there: A novel Perception and Attention Deficit model for recurrent complex visual hallucinations. Behavioral and Brain Sciences (2005) 28:6, pp. 737-757.

Collins, D.F., Cameron, T., Gillard, D. M., and Prochazka, A. (1998). Muscular sense is attenuated when humans move. Journal of Physiology, 508, 635-643.

Damasio, A. (1999). The Feeling of What Happens. Body and Emotion in the Making of Consciousness. New York: Harcourt Brace. 
David, N., Newen, A., Vogeley, K. (2008). The "sense of agency" and its underlying cognitive and neural mechanisms. Consciousness and Cognition, 17, 523-534

Feinberg, I. (1978). Efference copy and corollary discharge: Implications for thinking and its disorders. Schizophrenia Bulletin, 4, 636-640.

Ferrari, P.F., Bonini, L., and Fogassi, L. (2009). From monkey mirror neurons to primate behaviours: Possible 'direct' and 'indirect' pathways. Philos. Trans. R. Soc. Lond. B Biol. Sci., 364, 2311-2323.

Fourneret, P., de Vignemont, F., Franck, N., Slachevsky, A., Dubois, B., and Jeannerod, M. (2002). Perception of self-generated action in schizophrenia. Cogn. Neuropsychiatry, 7, 139156.

Fried, I., Mukamel, R., Kreiman, G., (2011). Internally Generated Preactivation of Single Neurons in Human Medial Frontal Cortex Predicts Volition. Neuron, 69, 548-562.

Frith, C.D. (1992). The cognitive neuro-psychology of schizophrenia. Hove, UK: Lawrence Erlbaum.

Frith, C.D. (2000). Blakemore, S., and Wolpert, D., Explaining the symptoms of schizophrenia: Abnormalities in the awareness of action. Brain Research Reviews, 31, 357-363.

Haggard, P. (2008). Human volition: towards a neuroscience of will. Nature Neoroscience Reviews, 8, 934-946.

Hawkins, R.D., and Kandel, E.R. (1984a). Is There a Cell-Biological Alphabet for Simple Forms of Learning? Psychological Review, 91, 375-391.

Hawkins, R.D., and Kandel, E.R. (1984b). Steps Toward a Cell-Biological Alphabet for Elementary Forms of Learning. In: G. Lynch, J.L. McGaugh, and N.M. Weinberger (eds.), Neurobiology of Learning and Memory (pp. 385-404) Guilford Press, New York.

Hindriks, K., Wiggers, P., Jonker, C.M. and Haselager, W. (2011). Towards A Computational Model of the Self-Attribution of Agency. In: Proc. of the 24th Intern. Conf. on Industrial, Engineering and Other Applications of Applied Intelligent Systems, IEA/AIE'11, Part I. Lecture Notes in AI, vol. 6703 (pp. 295-305). Springer Verlag.

Hopfield, J.J. (1982). Neural networks and physical systems with emergent collective computational properties. Proc. Nat. Acad. Sci. (USA), 79, 2554-2558.

Hopfield, J.J. (1984). Neurons with graded response have collective computational properties like those of two-state neurons. Proc. Nat. Acad. Sci. (USA), 81, 3088-3092.

Iacoboni M. (2008a). Mirroring People: the New Science of How We Connect with Others. Farrar, Straus \& Giroux.

Iacoboni, M. (2008b). Mesial frontal cortex and super mirror neurons. Behavioral and Brain Sciences, 31, 30-30.

Iacoboni, M., and Dapretto, M., (2006). The mirror neuron system and the consequences of its dysfunction. Nature Reviews Neuroscience, 7, pp 942-951.

Jeannerod, M. (2009). The sense of agency and its disturbances in schizophrenia: a reappraisal Exp Brain Res, 192, 527-532.

Keysers, C., Gazzola, V. (2010). Social Neuroscience: Mirror Neurons Recorded in Humans. Current Biology, 20, 253-254. 
Moore, J., and Haggard, P. (2008). Awareness of action: Inference and prediction. Consciousness and Cognition, 17,136-144.

Mukamel, R., Ekstrom, A.D., Kaplan, J., Iacoboni, M., and Fried, I. (2010). Single-Neuron Responses in Humans during Execution and Observation of Actions. Current Biology, 20, 750-756.

Samsonovich, A.V. (2005) Hallucinating objects versus hallucinating Subjects. Behavioral and Brain Sciences (2005) 28:6, pp. 772-773.

Schippers, M.B., Keysers, C. (2011). Mapping the flow of information within the putative mirror neuron system during gesture observation. Neuroimage, 57, 37-44.

Schutz-Bosbach, S., Avenanti, A., Aglioti, S.M., and Haggard, P. (2009). Don't Do It! Cortical Inhibition and Self-attribution during Action Observation. Journal of Cognitive Neuroscience, 21, 1215-1227.

Synofzik, M., Thier, P., Leube, D.T., Schlotterbeck, P., and Lindner, A. (2010). Misattributions of agency in schizophrenia are based on imprecise predictions about the sensory consequences of one's actions. Brain, 133, 262-271.

Treur, J., (2011). A Cognitive Agent Model Incorporating Prior and Retrospective Ownership States for Actions. In: Walsh, T. (ed.), Proc. of the Twenty-Second International Joint Conference on Artificial Intelligence, IJCAI'11, pp. 1743-1749.

Treur, J., and Umair, M., (2011). A Cognitive Agent Model Using Inverse Mirroring for False Attribution of Own Actions to Other Agents. In: Proc. of the 24th Intern. Conf. on Industrial, Engineering and Other Applications of Applied Intelligent Systems, IEA/AIE'11. Lecture Notes in Artificial Intelligence, vol. 6704 (pp. 109-119). Springer Verlag.

Voss, M., Moore, J., Hauser, M., Gallinat, J., Heinz, A., and Haggard, P. (2010). Altered awareness of action in schizophrenia: a specific deficit in predicting action consequences. Brain, 133, 3104-3112.

Weiskrantz, L., Elliot, J., and Darlington, C. (1971). Preliminary observations of tickling oneself. Nature, 230, 598-599.

Wolpert, D.M. (1997). Computational approaches to motor control. Trends in Cognitive Sciences, $1,209-216$. 